\title{
Zum 80. Geburtstag von Professor Horst Wagner
}

\section{Wolfgang Pöhl}

\section{Salzburg, Österreich}

Online publiziert 8. Januar 2020

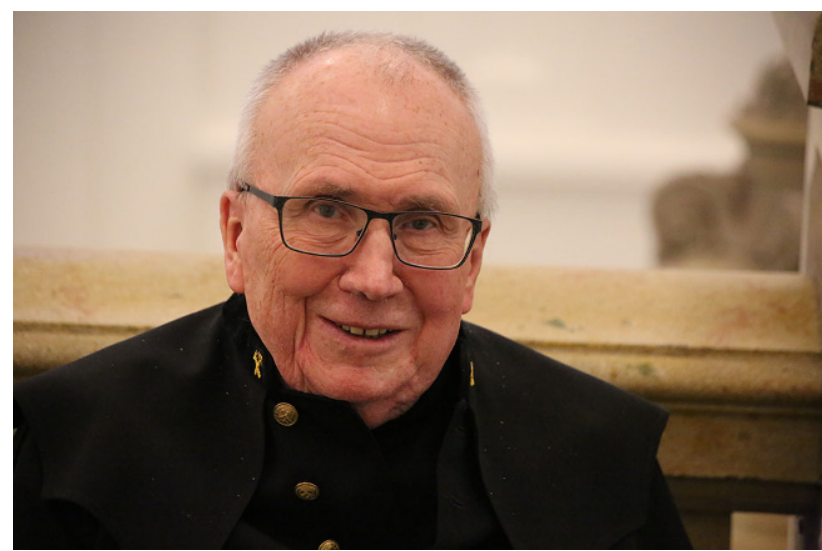

Im Sommer hat mich Horst Wagner gefragt, ob ich beim Rohstoffkolloquium 2019 anlässlich seines 80. Geburtstags eine Laudatio halten könne. Das war mir nicht nur Ehre, sondern auch große Freude, kennen wir beide uns doch seit dem 15. Lebensjahr und sind wir uns in all den Jahren immer wieder auf verschiedenen Ebenen begegnet. Horst Wagner hat ein so würdiges Alter erreicht, dass ob seiner außerordentlichen Leistungen bereits zahlreiche Laudationen in Südafrika und Österreich gehalten wurden, aus denen ich schöpfen konnte. So war es mir nicht nur Freude, sondern ein Wunsch, diese Laudatio zu halten.

Horst wurde am 24. Mai 1939 in Salzburg geboren. Von der Hauptschule wechselte er mit 14 Jahren in die Realschule Salzburg, wo wir uns vor allem auf sportlicher Ebene kennen lernten. Nach der Matura 1957 wählte er den verlängerten Wehrdienst bei den Funkern und wollte eine Offizierskarriere anstreben, musste aber erkennen, dass er die gewünschte Ausbildung nicht bekommen konnte. Er rüstete 1958 wieder ab und trat bis zum Beginn des Studiums 1959 für 9 Monate die Stelle eines Lehrhauers am Kupferbergbau Mitterberg - Mühlbach-Hochkönig an. Ein Freund der Familie hat ihm zu dieser glücklichen Entscheidung verholfen. Horst inskribierte an der Montanistischen

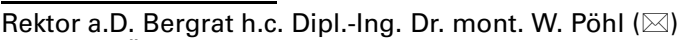
Salzburg, Österreich wolfgangpoehl@gmx.at
Hochschule Leoben das Studium Bergbau und Markscheidewesen. Ich folgte inm nach einer abgebrochenen Militärkarriere bei den Pionieren mit denselben Studienrichtungen nach. Zwei wesentliche Punkte waren für unser beider Entscheidung für die Montanistische Hochschule, wie sie früher geheißen hat, ausschlaggebend: Beide mussten wir für unser Studium selbst aufkommen und es war die Persönlichkeit Fettweis, die uns mit seinem Auftreten imponiert und begeistert hat. Wir wussten, 300 Schichten Praktikum und die Möglichkeit, als wissenschaftliche Hilfskraft an der Hochschule zu arbeiten, finanziert fast spielend ein montanistisches Studium.

Prof. Fettweis verlieh der Uni eine gewisse Modernität und setzte sich mit enormem Bemühen für eine Internationalität des Fachbereichs ein, und das in einer Zeit, wo der Bergbau in Europa zunehmend in Schwierigkeiten kam.

Horst Wagner hat das Studium im Eiltempo im Winter 1963 als Diplomingenieur beendet. Von 1964 bis 1969 war Horst Wagner Hochschulassistent bei Prof. Fettweis. In dieser Zeit konnte er uns Studierende bei den Übungen begeistern. Das Schwergewicht lag bei der Fels- und Gebirgsmechanik und Wettertechnik, bis zu diesem Zeitpunkt forschungsmäßig im Europäischen Bergbau noch mäßig besetzt.

1968 wurde Horst Wagner mit der Doktorarbeit „Photoelastische Untersuchungen über die Vorgänge "beim Schneiden von Gestein“ und einem mit Auszeichnung bestandenen Rigorosum zum Doktor der montanistischen Wissenschaften promoviert. Das Mekka der Felsmechanik war zu diesem Zeitpunkt Südafrika. Schon während seiner Doktorarbeit gab es Kontakte mit Südafrika; schließlich war es Fettweis, der die erste Adresse vermittelte.

Horst Wagner wanderte 1969 nach Südafrika aus, mit inm seine Frau Annelore, die er 1966 in der DDR in Magdeburg kennen gelernt hatte und die leider im November 2019 verstorben ist.

Horst Wagners besonderes Hobby, das er seit der Schulzeit mit Erfolg betreibt, ist der Modellfreiflug. Er wurde mehrfacher österreichischer Staatsmeister und war auch international sehr erfolgreich. Dieses Hobby hat inn mit seiner Frau zusammengeführt. In der DDR haben sie am 21.07.1967 geheiratet. Die spätere Ausreise von Annelore 
aus der DDR ist im darauf folgenden September gelungen, nachdem Horst Wagner als Hochgebirgemontanist in der DDR, mangels hoher Berge, nicht beschäftigt werden konnte. Bis zu diesem Zeitpunkt durfte Annelore wegen Fluchtgefahr nur dann mit dem Segelflugzeug fliegen, wenn der Wind sie nicht in den Westen getragen hätte.

Mit Katharina, die 1968 in Leoben geboren wurde, ging es dann nach Südafrika, Sohn Alexander wurde 1972 dort geboren. In Johannesburg startete Horst eine unglaubliche 25-jährige Karriere. Der Bogen reicht vom Nachwuchswissenschafter bis zur Ernennung zum Generaldirektor der Chamber of Mines Research Organisation 1986. In dieser Funktion war Wagner im Bereich Forschung für 9 Forschungslabors und 650 Mitarbeiter verantwortlich. Horst Wagners Fähigkeiten waren so hervorragend und bahnbrechend, dass er von 1988 bis 1994 die Position des Senior General Manager - Operations (Chamber of Mines) innehatte. Er war verantwortlich für die Bereiche Forschung, Technologie, Bergbausicherheit und Umwelt, Unfallschutzversicherung, Goldraffinerie und Uranverarbeitung und Vermarktung. Überraschend war diese Führungsposition für Horst Wagner insofern, da er als Nicht-Südafrikaner eine Position erhielt, die sich die mit ihm arbeitenden Südafrikaner erhofft hatten. Der Board der Chamber of Mines entschied sich für inn mit den Worten: „Horst, this is all yours." Horst Wagner hat mit viel Begeisterung in dieser Position gewirkt und hat höchste Anerkennung erlangt. Daher war die Entscheidung, nach Österreich zurückzukehren, nicht leicht, aber da gab es Ereignisse, die für diese Entscheidung sprachen.

Die sehr intensiven internationalen Aktivitäten haben immer wieder ermöglicht, Horst Wagner zu treffen und eine fachlich-freundschaftliche Beziehung aufrecht zu halten. So war Horst Wagner Vorsitzender des südafrikanischen Nationalkomitees für den Weltbergbaukongress, in dem er von 1985 bis 1994 Südafrika vertreten hat. Angeführt von Prof Fettweis, war ich Mitglied des österreichischen Komitees des Weltbergbaukongresses. Vor allem sind die Beziehungen von Wagner zu Prof. Fettweis in all den Jahren aufrecht gewesen. Fettweis hat Wagner auch bei seinen Reisen ins südliche Afrika mehrmals zum Gedankenaustausch besucht. Fettweis hat es auch in die Wege geleitet, dass Wagner im Mai 1992 zum korrespondierenden Mitglied der Akademie der Wissenschaften gewählt wurde. Mit der nahenden Emeritierung von Prof. Fettweis im Jahr 1994 begann im Jahr davor die große Suche nach einem würdigen Nachfolger, und der Wunschkandidat war Horst Wagner. Zufällig war ich 1993 im Auftrag meines Unternehmens in Johannesburg, und es war eine Selbstverständlichkeit, sich im Hotel mit Horst zu treffen, sollte ich doch im Auftrag von Prof. Fettweis versuchen, Wagner für seine Nachfolge zu gewinnen. So ist auch das Gespräch gelaufen. Was ich nicht wusste war, dass Horst drei Tage zuvor überfallen worden war, was sich mit einem Einschussloch rechts vom Gaspedal seines Mercedes zeigte. Auch noch sichtbar in Leoben, da der Mercedes mit Rechtssteuerung nach Leoben übersiedelt wurde. Das war aus meiner Sicht ein unterstützendes Argument dafür, nach Österreich zu gehen. Erst bei unserem Gespräch im Oktober 2019 in Leoben konnte ich erfahren, dass dieser Vorfall nur einen kleinen Teil zu dieser seiner Entscheidung beigetragen hat. Hauptgrund war die geänderte politische Situation durch den ANC, die Annelore befürchten ließ, dass sie nochmals die DDR erleben müsse. Für Horst Wagner hat sich eine neue Situation dadurch ergeben, dass seine Position in vier Chief Executive-Positionen aufgeteilt wurde. Auf diese Weise wollte die Chamber of Mines eine zukünftige schwarze Regierung, sprich Verstaatlichung, verhindern oder zumindest erschweren. So wurde von der Familie Wagner die Entscheidung getroffen: Wir gehen nach Leoben. Was uns Montanisten sehr glücklich machte.

Am 1. März 1994 nahm Professor Horst Wagner seine Tätigkeit als ordentlicher Professor für Bergbaukunde, Bergtechnik und Bergwirtschaft an der Montanuniversität auf. Im Jahr 2000 wurde Wagner wirkliches Mitglied der Akademie der Wissenschaften. Im Jänner 1995 wurde Wagner zum Vorsitzenden des Universitätskollegiums gewählt. Diese Funktion hat er bis zum Herbst 2001 ausgeübt. Von 2000 bis 2006 war Wagner Vizepräsident des Internationalen Organisationskomitees des Weltbergbaukongresses, wodurch ein internationaler Brückenschlag für den montanistischen Bereich gegeben war.

Es war Horst Wagner, der mich im Jahr 1998 ermunterte, mich für die Stelle des Rektors der Montanuniversität, die für die Jahre 1999-2003 ausgeschrieben war, zu bewerben. So kam es auch, dass wir uns gemeinsam um das Wohl der Montanuniversität kümmern konnten. In der Zeit als dessen Vorsitzender war Horst Wagner mit dem Universitätskollegium entscheidend für die Umsetzung der Wachstumsstrategie der MUL mitverantwortlich. Es ging vor allem um eine verstärkte Profilbildung der Montanuniversität innerhalb der österreichischen Universitäten. Es gab damals Ambitionen, die MUL auf die Universitäten Graz und Linz aufzuteilen. Im Rahmen eines Strategieprozesses wurden die Schwerpunkte der Montanuniversität herausgearbeitet. Als Ergebnis dieses Prozesses wurde die "3M“ Gruppe Mining-Metallurgy-Materials, ergänzt durch Montanmaschinenwesen, geprägt. Ich möchte es nicht versäumen, mich an dieser Stelle auch beim zweiten Geburtstagskind Prof. Moser zu bedanken, der bei diesem Strategieprozess mit viel Einsatz dabei war.

Was sind die großen Forschungs- und operativen Leistungen von Prof. Horst Wagner? Besonders bahnbrechend und von großer praktischer Auswirkung waren seine Untersuchungen auf dem Gebiet des Grubenausbaus und der Gebirgsmechanik zur Beherrschung und Vermeidung von Gebirgsschlägen. Dadurch kam es zu weniger durch Gebirgsschläge verursachten tödlichen Unfällen. Horst Wagner war bei seinen Entscheidungen nie blind durch Forschungsergebnisse geleitet, er hatte immer ein wachsames Auge auf das Gebirge, so auch bei der Bemessung von Hohlräumen für das jeweilige Abbauverfahren. Insbesondere war es die Fähigkeit von Horst Wagner, anwendungsbezogene Forschungsergebnisse so weiter zu entwickeln, dass sie auch unter wechselhaften Bedingungen nutzbar gemacht werden konnten. In Österreich verschob sich der Schwerpunkt seiner Tätigkeiten zunehmend auf die Probleme der Versorgung mit mineralischen Rohstoffen. Dieses Problemfeld wurde zum internationalen Thema. 
Wagner hat zwei EU-Projekte bearbeitet und es kam zu einer sehr intensiven weltweiten Zusammenarbeit mit vieIen wissenschaftlichen Instituten. Besonders ist hier das sogenannte "Leoben Paper" zu nennen, eine Studie über die Versorgung Europas mit nicht-energetischen mineralischen Rohstoffen, die ein europäisches Konsortium unter Leitung von Prof. Wagner erarbeitete. Unvergesslich der Einsatz von Horst Wagner vier Tage nach dem schrecklichen Grubenunglück von Lassing am 17. Juli 1997. Horst Wagner hat sich freiwillig als Leiter des Rettungswerkes, das zu diesem Zeitpunkt mit Problemen behaftet war, zur Verfügung gestellt. Seine südafrikanischen Erfahrungen, die Horst einbringen konnte, haben nach 9 Tagen die Rettung des Bergmanns Heinzl möglich gemacht.

Nach seiner Emeritierung am 1. Oktober 2007 hat Horst Wagner seine Tätigkeit bis heute fast uneingeschränkt fortgeführt. Er hält nach wie vor Vorlesungen an der MUL, betreut Diplom- und Doktorarbeiten, berät und betreut Unternehmen, ist als Aufsichtsrat tätig, erweiterte seine mehr als 230 Veröffentlichungen um weitere Beiträge und nimmt auch sein Hobby des Modellfreifluges weiterhin vom Rollstuhl aus wahr.

Dieser Rollstuhl ist die Folge eines unglücklichen Sturzes durch Benommenheit in einem Hotel in Argentinien, wo Horst Wagner an der Weltmeisterschaft des Modellfreifluges im Mai des Jahres 2005 teilgenommen hat. Nur dank der Fürsorge seiner ihn begleitenden Gattin hat er diesen Unfall überlebt. Während seiner monatelangen Unfallbehandlungen in Österreich hat das Schicksal wieder zugeschlagen, Annelore erlitt einen Schlaganfall, der sie mehr als Horst an den Rollstuhl gefesselt hat. Die Tochter, die in Graz Kunst studiert hatte, betreute ihre Eltern zu Hause bis zum Tod von Annelore. Der Sohn Alexander studierte Physik, lebt mit seiner Familie in Südafrika und hat seine Eltern mit zwei Enkelkindern glücklich gemacht.

Die Stärke Horst Wagners ist es, Probleme ganzheitlich zu sehen und das zu machen, was man noch kann. Er meinte: „Ich habe keine andere Option." Der Rollstuhl hindert nicht daran, Unternehmen wie Boliden-Kiruna, auch die Bergbaue der RHI vor Ort zu beraten. Es ist daher nicht von ungefähr, dass Horst Wagner viele Ehrungen erfahren hat. Eine besondere Ehrung in Südafrika durch Dr. Barza, Vorstand des South African Institute of Mining and Metallurgy, mit der Verleihung des Brigadier Stokes Memorial Award mit den Worten: „We pay homage to a leader of greatness and a man of great humility." Nicht anders bei der Verleihung der höchsten Auszeichnung des österreichischen Bergbaues, der Miller-von-Hauenfels-Medaille im Mai 2007. Sektionschef Dr. Alfred Maier schließt mit den Worten: „Ein Bergmann mit Anstand, Mut und Verstand, unglaublich fleißig und verlässlich, immer um einen positiven Beitrag bemüht."

Horst, danke, du hast uns viel erleben lassen und gegeben und gibst immer noch. Wir hoffen, dass es noch lange so bleibt. Man könnte zum Wagnerianer werden.

Alles Gute

Glückauf!
Quellen Persönliche Begegnungen mit Horst Wagner G. B. L. Fettweis, P. Moser, BHM 154 (2009), H. 6 Wikipedia: Horst Wagner (Montanwissenschafter) H. Phillips: A tribute to Emeritus Horst Wagner, BHM 160 (2015), H. 4

Die wesentlichen beruflichen Tätigkeiten von 0 . Univ.-Prof. Horst Wagner 1964-1958: Assistent am Institut für Bergbaukunde, Bergtechnik und Bergwirtschaft, Montanistische Hochschule Leoben

1969-1994: Chamber of Mines of South Africa. 1969 Senior Research Officer, 1972 Chief of Rock Mechanics Division, 1977 Director (Mining, Operations, Laboratory), $1986 \mathrm{Di}-$ rector General, 1994 Senior General Manager (Forschung, Technologie, Bergbausicherheit und Umwelt, Unfallschutzversicherung, Goldraffinerie und Uranverarbeitung und Vermarktung)

1994-2007: Ordinarius für Bergbaukunde, Bergtechnik und Bergwirtschaft (Montanuniversität Leoben)

2007-dato: Emeritierter o. Univ.-Prof. für Bergbaukunde, Bergbautechnik und Bergwirtschaft

Berufsbezogene Aktivitäten 1977-1988: Vorsitzender der Südafrikanischen Gesellschaft für Gebirgsmechanik

1984-1986: Vizepräsident, später Präsident des "South African Institute of Mining and Metallurgy"

1986-1988: Mitglied des "National Science Advisory Council“ der Republik Südafrika

1986-1994: Honorarprofessor für Bergbauwissenschaften an der Witwatersrand-Universität, Johannesburg

2000-dato: Wirkliches Mitglied der österreichischen Akademie der Wissenschaften, 2003 Obmann der Kommission für Grundlagen der Mineralstoffforschung

1994-2007: Vorsitzender der Studienkommission für Bergwesen

1995-2001: Vorsitzender des Universitätskollegiums 2002-2006: Mitglied des Senats der Montanuniversität 1995-1999: Vizepräsident des BVÖ, bis 2003 Präsident

2000-2006: Vizepräsident des Internationalen Organisationskomitees des Weltbergbaukongresses

2004-dato: Stellvertretender Vorsitzender des Stiftungsbeirates der VA-Erzberg Privatstiftung und des Aufsichtsrates der VA-Erzberg GmbH

Ehrungen und Auszeichnungen 1988: Gold Medal - South African Institute of Mining and Metallurgy

1994: Brigardier Stokes Memorial Award

1999: Ehrenkreuz für Wissenschaft und Kunst 1. Klasse

2003: Lifetime Award - Südafrikanische Gesellschaft für Gebirgsmechanik

2004: Ehrenzeichen des Fachverbandes der Wirtschaftskammer; Ehrenmitglied der Ungarischen Akademie der Wissenschaften

2007: Miller-von-Hauenfels-Medaille des Bergmännischen Verbandes; Großes Ehrenzeichen in Silber der Republik Österreich; Großes Ehrenzeichen des Landes Steiermark

2013: Verleihung des Berufstitels „Bergrat h.c."

Wolfgang Pöhl 